\title{
Diversity of Cooperation in the Tragedy of the Commons
}

\author{
Timothy Killingback \\ Department of Mathematics \\ University of Massachusetts Boston \\ Boston, MA, USA \\ timothy.killingback@umb.edu
}

\author{
Michael Doebeli \\ Department of Mathematics \\ University of British Columbia \\ Vancouver, BC, Canada \\ doebeli@math.ubc.ca \\ \& \\ Department of Zoology \\ University of British Columbia \\ Vancouver, BC, Canada

\section{Christoph Hauert} \\ Department of Mathematics \\ University of British Columbia \\ Vancouver, BC, Canada \\ hauert@math.ubc.ca
}

Common-pool resources are of fundamental importance in biology and the social sciences. The use of a shared limited resource is an exemplar of a situation in which individuals can behave cooperatively, through modest consumption, or selfishly, through excessive consumption (Hardin 1968, 1998; Berkes et al. 1989; Ostrom et al. 1999; Ostrom 1999; Frank 1989; Foster 2004). The essential paradox concerning the use of such common-pool resources is paraphrased by Hardin's celebrated Tragedy of the Commons (Hardin 1968): each individual benefits from consuming the resource, but the incurring costs are diluted and shared among all interacting individuals. Hence the common resource is prone to exploitation and self-interest drives over-consumption of the resource to the detriment of all. Here we show evolutionary outcomes based on a continuous range of consumption levels of common-pool resources that are often strikingly different from the classic Tragedy of the Commons. In fact, a second tragedy is revealed: not only is the common resource overexploited, but selection may result in states in which high and low consumers stably coexist. In a different, but related context this has been termed the Tragedy of the Commune, according to which evolution in communal enterprises may favor mixed states with restraint and excessive individuals rather than egalitarian consumption levels (Doebeli 2004). At least in human societies this clearly runs against accepted notions of fairness and bears formidable risks for escalating conflicts.

Public goods play an important role in biology and human society. All organisms depend on common-pool nutrients for their survival and complex human societies depend both on natural resources such as fossil fuels, the global atmosphere, or the world's fisheries, as well as on man-made resources, such as social welfare or the Internet (Ostrom et al. 1999; Milinski et al. 2006). Such public resources benefit everyone but the costs of abuse are shared by all or even deferred to future generations. Therefore, excessive consumption is cheap for the individual but costs the community dearly. The classical Tragedy of the Commons (Hardin 1968, 1998) states that the shared and unregulated use of limited resources is bound to result in overexploitation despite the fact that this puts everybody in a worse position (Berkes et al. 1989; Frank 1998; Ostrom 1999; Ostrom et al. 1999).

The Continuous Snowdrift game (Doebeli et al. 2004) describes the complementary situation in which individuals make costly contributions to a public good. The benefit that accrues to all interacting individuals is determined by their accumulated contributions. Thus, the individual determines the costs and the community shares the benefits as opposed to the Tragedy of the Commons, in which individuals specify their consumption while the community shares the costs. One possible evolutionary outcome in the Continuous Snowdrift game is diversification in levels of contribution. This leads to coexistence between highly cooperative individuals that make large contributions to the public good, and defectors that make little or no contributions. This outcome was termed the Tragedy of the Commune (Doebeli et al. 2004) because in human societies 
large differences in contributions to communal efforts may not be tolerated. Here we demonstrate that such behavioral diversification and the accompanying social tensions also occur in the Tragedy of the Commons, where individuals consume a common resource instead of contributing to a public good.

In the Tragedy of the Commons, the evolution of quantitative traits determining exploitation of common resources can be analyzed using evolutionary game theory (Maynard Smith 1982). Consider $N$ individuals that share a common resource, with individual $i$ consuming an amount $x_{i} \geq 0(i=1, \ldots, N)$. Each individual benefits from consuming the resource, but because the resource is common and finite, the incurring costs depend on the total consumption of all individuals. Costs are low if the overall consumption levels are low and the public resource can be sustained, but if the resource is overexploited the associated costs increase. For example, if fish stocks are depleted, the costs per catch are augmented. The simplest expression for the payoff to an individual $i$ consuming $x_{i}$ in a group of $N$ individuals consuming $\bar{x}=\left(x_{1}, x_{2}, \ldots, x_{N}\right)$ is given by $P\left(x_{i} ; \bar{x}\right)=B\left(x_{i}\right)-C\left(x_{1}+\cdots+x_{N}\right)$. In this expression $B(x)$ and $C(x)$ are benefit and cost functions, which determine the gain of individual $i$ due to its own consumption, and its loss due to the accumulated consumption of all individuals, respectively. Here we assume that $B(x)$ and $C(x)$ are smooth and strictly increasing functions, with $B(0)=C(0)=0$ such that no consumption returns no benefit and incurs no costs. We also assume that, at least for small $x, B(x)>C(x)$. This simply reflects the fact that the public resource is valuable and thus creates an incentive for individuals to consume and take advantage of the resource. Naively, we might expect that this condition promotes nonvanishing consumption levels of all individuals. The following analysis demonstrates that the evolutionary dynamics is much richer and, in particular, may lead to evolutionarily stable outcomes where modest and reckless consumers can coexist.

The consumption level $x_{i}$ of each individual is a continuous trait, and evolutionary changes of the consumption level can be studied using adaptive dynamics (Geritz et al. 1998). The underlying assumption is that a resident population exists that is monomorphic in trait $x$. The growth rate of a rare mutant trait $y$ is then given by the invasion fitness $f_{x}(y)=P(y ; \bar{x})-P(x ; \bar{x})$, where $\bar{x}=(x, \ldots, x)$ because the resident population is monomorphic in $x$. Note that rare mutants do not affect the payoff of the resident. The adaptive dynamics of the resident trait $x$ is governed by the selection gradient $D(x)=\partial f_{x}(y) /\left.\partial y\right|_{y=x}=B^{\prime}(x)-C^{\prime}(N x)$, with $x$ obeying the equation $\dot{x}=\mu D(x)$, where $\mu$ is a parameter describing how mutations influence the speed of evolution. In the following, we assume without loss of generality that $\mu=1$. Selection favors individuals with higher (lower) levels of consumption if $D(x)>0(D(x)<0)$. Trait values $x^{*}$ at which the selection gradient vanishes, $D\left(x^{*}\right)=0$, are called singular points. If no solutions of $D\left(x^{*}\right)=0$ exist, then the trait $x$ keeps monotonically increasing $(D(x)>0)$ or decreasing $(D(x)<0)$. A singular trait $x^{*}$ is convergence stable if $d D(x) /\left.d x\right|_{x=x^{*}}=B^{\prime \prime}\left(x^{*}\right)-N C^{\prime \prime}\left(N x^{*}\right)<0$, i.e., represents an attractor for the adaptive dynamics. If this inequality is reversed, then $x^{*}$ is a repellor and traits evolve away from $x^{*}$.

At a singular trait $x^{*}$ the classical Tragedy of the Commons is recovered - all individuals in the population uniformly overconsume the resource to their mutual detriment. This follows from observing that at $x^{*}$ each individual receives a payoff $B\left(x^{*}\right)-C\left(N x^{*}\right)$; but if all individuals reduced their consumption by a small amount $\epsilon>0$ to $x^{*}-\epsilon$, then, to first order in $\epsilon$, each individual's payoff changes by $(N-1) B^{\prime}\left(x^{*}\right) \epsilon$, as can be seen from a Taylor expansion of the payoff function around $x^{*}$ and using $B^{\prime}\left(x^{*}\right)=C^{\prime}\left(N x^{*}\right)$. This indicates an improvement because $B(x)$ is a strictly increasing function. Hence, a uniform reduction in the consumption level by $\epsilon$ results in an increased payoff for all. Therefore, as predicted by the Tragedy of the Commons, at $x^{*}$ the population is overconsuming the resource at a loss to all.

However, even if the trait $x^{*}$ is convergent stable, it does not necessarily represent an evolutionary end state. Generically, a convergent stable singular strategy $x^{*}$ represents either a maximum or minimum of the invasion fitness (Geritz et al. 1998). If $x^{*}$ is a maximum, i.e., $\partial^{2} f_{\bar{x}^{*}}(y) /\left.\partial y^{2}\right|_{y=x^{*}}<0$, then $x^{*}$ is evolutionarily stable and cannot be invaded by any mutant (see Figure 1(a)). Resident traits in the vicinity of $x^{*}$ converge to $x^{*}$ and the Tragedy of the Commons applies. If, however, $x^{*}$ indicates a minimum, i.e., $\partial^{2} f_{\bar{x}^{*}}(y) /\left.\partial y^{2}\right|_{y=x^{*}}>0$, then a resident population that is monomorphic for $x^{*}$ can be invaded by mutants with trait values on either side of $x^{*}$ (Geritz et al. 1998). In this case, the point $x^{*}$ is called an evolutionary branching point, and after converging to this point, the population spontaneously splits into two distinct and diverging phenotypic clusters, as illustrated in Figure 1(b). For the costs and benefits in the Tragedy of the Commons, evolutionary instability of the singular point occurs if $B^{\prime \prime}\left(x^{*}\right)-C^{\prime \prime}\left(N x^{*}\right)>0$. After evolutionary branching, evolution leads to stable coexistence of modest and excessive consumers (Figure 1(b)). Using similar arguments as in the case of a population that is monomorphic for the singular trait, it could be possible to show that this coexistence equilibrium also represents a Tragedy of the Commons: the common resource is overexploited in the sense that if all the excessive consumers would lower their consumption, the payoff of all individuals in the population would increase. We also note that at the coexistence equilibrium, the two strategies present, modest and excessive consumers, play a classical Snowdrift game (Sugden 1986; Hauert et al. 2006) so that either strategy could invade a population consisting of individuals having the other strategy.

Evolutionary branching occurs whenever $x^{*}$ is convergent stable and evolutionarily unstable, i.e., whenever 

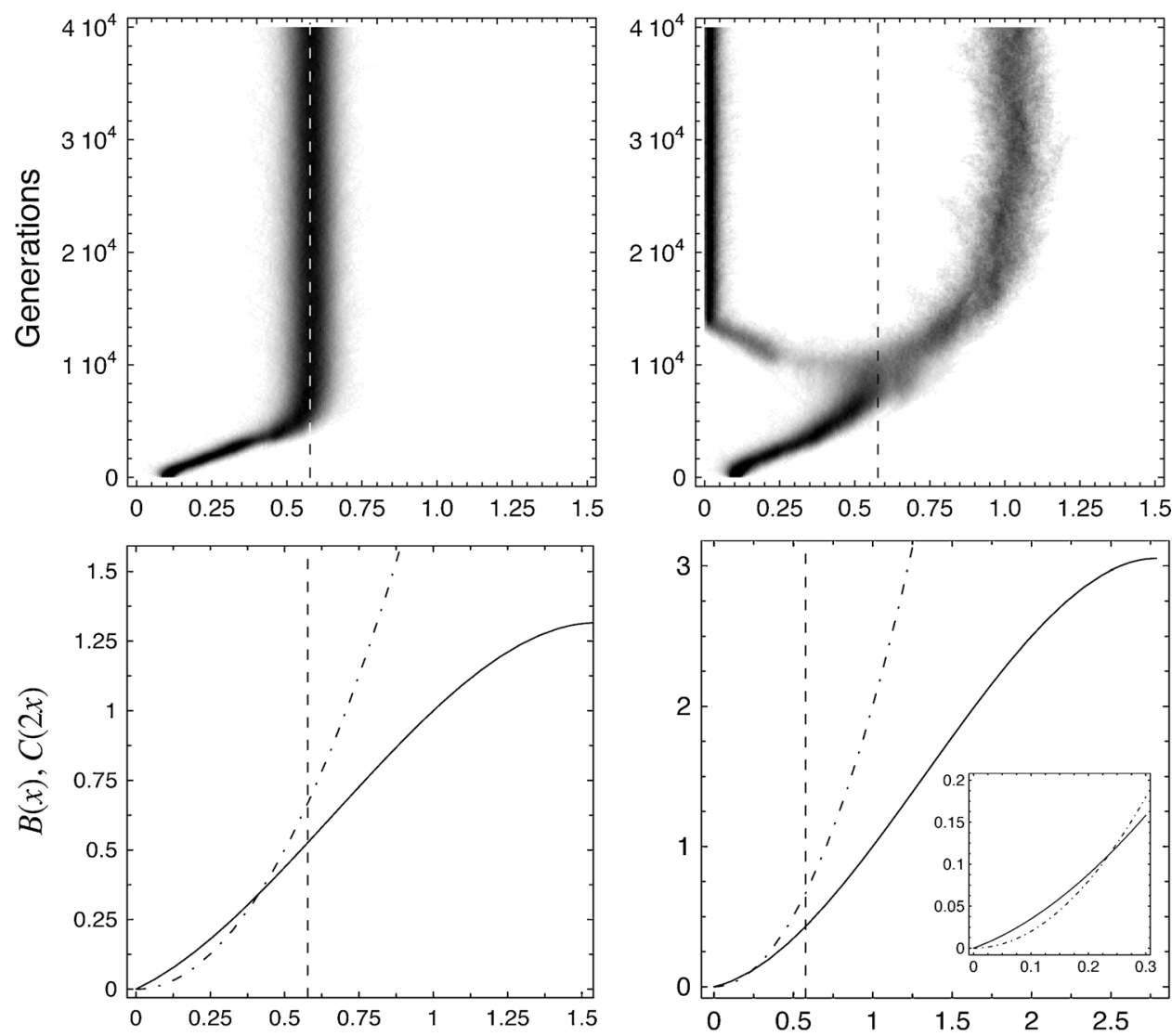

(a)

Trait

(b)

Trait

Figure 1.

Evolutionary dynamics of Hardin's Tragedy of the Commons with a continuous range of consumption levels of the common resource (a) for stable intermediate consumption levels and (b) for phenotypic diversification into moderately and excessively consumers. The evolution of the trait distribution (top row) is illustrated for two distinct scenarios together with the corresponding benefit (solid line) and cost functions (dash-dotted line) in homogeneous populations (bottom row). Darker shades indicate higher trait densities and the dashed lines mark the singular trait $x^{*}$. In both the cases, the benefits exceed the costs at low trait values (see inset). (a) If $x^{*}$ is convergent stable and evolutionarily stable, the dynamics leads to a homogenous, egalitarian state of intermediate consumption levels. (b) Conversely, if $x^{*}$ is convergent stable but not evolutionarily stable, evolutionary branching occurs and the population splits into two distinct phenotypic clusters of modest and excessive consumers. This links the Tragedy of the Commons to the Tragedy of the Commune (Doebeli et al. 2004). Technical details: $B(x)=-a x^{3}+2 b x^{2}+a x, C(x)=b x^{2}$ with $b=0.5$ and (a) $a=0.25$ and (b) $a=0.5 ; N=2$; population size 10,000; Gaussian mutations, rate 0.01 , standard deviation \pm 0.005 .

$N C^{\prime \prime}\left(N x^{*}\right)>B^{\prime \prime}\left(x^{*}\right)>C^{\prime \prime}\left(N x^{*}\right)$. Thus, for evolutionary branching the cost function must be concave at $N x^{*}$ and the benefit function at $x^{*}$. Realistic cost and benefit functions often satisfy these conditions. Accelerating costs represent a natural assumption and are often observed (Siby and McFarland 1976; Killingback and Doebeli 2002). Similarly, benefits also often accelerate initially and then saturate, as in the case of Sigmoid benefit functions (Parker et al. 1972; Maynard Smith 1982). A suitable choice of cost and benefit functions permits a full analysis of the dynamics. For example, the functions $B(x)=-a x^{3}+2 b x^{2}+a x$ and $C(x)=b x^{2}$ with $a, b>0$ satisfy our assumptions in the trait interval $x \in\left[0,\left(2 b+\sqrt{4 b^{2}+3 a^{2}}\right) /(3 a)\right]$. For pairwise interactions, $N=2$, this admits a unique and parameter-independent singular point at $x^{*}=1 / \sqrt{3}$ that is always convergent stable, $\sqrt{3} a / b>0$. The singular trait marks an evolutionarily stable state if $\sqrt{3} a / b>1$ and a branching point otherwise. The two scenarios are depicted in Figure 1 and can be further explored using the VirtualLabs (Hauert 2010).

The evolution of continuous levels of consumption in the Tragedy of the Commons is a complement to the continuous levels of investment in the Snowdrift game (Doebeli et al. 2004). The Continuous Snowdrift game describes a situation in which individuals, instead of consuming a common resource, make contributions to a public good. In this case, the benefit is an increasing function of the sum of all contributions made by interacting individuals, whereas the cost is a function of individual contributions. This game exhibits qualitatively very similar evolutionary dynamics as the Tragedy of the Commons, albeit under "dual" conditions. In particular, in the Continuous Snowdrift game evolutionary branching can only occur if the cost and benefit functions have negative curvatures at a singular 
point (Doebeli et al. 2004), while evolutionary branching in the Tragedy of the Commons requires positive curvatures.

In the Tragedy of the Commons, the public resource is exploited either in an egalitarian fashion where everybody equally overconsumes (if $x^{*}$ is evolutionarily stable) or through diversification into excessive and restrained consumers (if $x^{*}$ is a branching point). At least in human societies, the latter situation is, in fact, worse because the unequal levels of consumption run against the accepted notion of fairness (Fehr and Gächter 2000; Nowak et al. 2000). This is further aggravated when dealing with emotionally charged topics in nature conservation such as protecting whales or rain forests, which may lead to frustration in modest consumers because of their inability to alleviate the situation and hence bears formidable risks of escalating conflicts or militant interventions. Thus, the Tragedy of the Commons may be compounded by social tensions reminiscent of the Tragedy of the Commune reported in the Continuous Snowdrift game (Doebeli et al. 2004). The Tragedy of the Commons applies whenever common resources are overexploited and we propose that the Tragedy of the Commune applies whenever evolution promotes inequalities among interacting individuals.

The finding of diversified consumption levels in the Tragedy of the Commons may have substantial implications for understanding how resources are shared in many systems. For example, it is generally believed that within-host competition among different pathogen types will often result in the pathogens evolving to greater virulence, corresponding to overconsumption of their common resource in accord with the Tragedy of the Commons (Brown et al. 2002). Our analysis suggests that instead, diversification into coexisting pathogen strains with high and low virulence might be a common aspect of many diseases. For human societies, our analysis could, for example, provide a basis for understanding discrepancies in the individual depletion of increasingly scarce natural resources.

\section{Acknowledgments}

C.H. and M.D. acknowledge support from the Natural Sciences and Engineering Research Council, Canada (NSERC).

\section{References}

Berkes F, Feeny D, McCay BJ, Acheson, JM (1989) The benefits of the commons. Nature 340: 91-93.

Brown S, Hochberg M, Grenfell B (2002) Does multiple infection select for raised virulence? Trends in Microbiology 10: 401-405.

Doebeli M, Hauert C, Killingback T (2004) The evolutionary origin of cooperators and defectors. Science 306: 859-862.

Fehr E, Gächter S (2000) Fairness and retaliation: The economics of reciprocity. Journal of Economic Perspectives 14(3): 159-181.

Foster KR (2004) Diminishing returns in social evolution: The not-so-tragic commons. Journal of Evolutionary Biology 17: 1058-1072.

Frank SA (1998) Foundations of Social Evolution. Princeton, NJ: Princeton University Press.

Geritz SAH, Kisdi E, Meszéna G, Metz JAJ (1998) Evolutionarily singular strategies and the adaptive growth and branching of the evolutionary tree. Evolutionary Ecology 12: 35-57.

Hardin G (1968) The tragedy of the commons. Science 162: 1243-1248.

Hardin G (1998) Extensions of "the tragedy of the commons." Science 280: 682-683.

Hauert C (2010) VirtualLabs: Interactive tutorials on evolutionary game theory. http://www.univie.ac.at/virtuallabs

Hauert C, Michor F, Nowak M, Doebeli M (2006) Synergy and discounting of cooperation in social dilemmas. Journal of Theoretical Biology 239: 195-202.

Killingback T, Doebeli M (2002) The continuous prisoner's dilemma and the evolution of cooperation through reciprocal altruism with variable investment. American Naturalist 160(4): 421-438.

Maynard Smith J (1982) Evolution and the Theory of Games. Cambridge, UK: Cambridge University Press.

Milinski M, Semmann D, Krambeck H-J, Marotzke M (2006) Stabilizing the earths climate is not a losing game: Supporting evidence from public goods experiments. Proceedings of the National Academy of Sciences of the USA 103: 3994-3998.

Nowak MA, Page KM, Sigmund K (2000) Fairness versus reason in the ultimatum game. Science 289: 1773-1775.

Ostrom E (1999) Governing the Commons. Cambridge, UK: Cambridge University Press.

Ostrom E, Burger J, Field CB, Norgaard RB, Policansky D (1999) Revisiting the commons: Local lessons, global challenges. Science 284: 278-282.

Parker G, Baker R, Smith V (1972) The origin and evolution of gamete dimorphism and the male-female phenomenon. Journal of Theoretical Biology 29: 135-162.

Siby R, McFarland D (1976) On the fitness of behavior sequences. American Naturalist 110: 601-617.

Sugden R (1986) The Economics of Rights, Co-operation and Welfare. Oxford and New York: Blackwell. 